\title{
On finite state automaton actions of HNN extensions of free abelian groups
}

\section{Prokhorchuk V.}

\begin{abstract}
HNN extensions of free abelian groups are considered. For arbitrary prime $p$ it is introduced a class of such extensions that act by finite automaton permutations over an alphabet $\mathrm{X}$ of cardinality $p$ and belong to $p$-Sylow subgroup of the group of automaton permutations over such $\mathrm{X}$. As a corollary it implies that all corresponding HNN extensions are residually $p$-finite.

Key words and phrases: automaton group, automorphism of rooted tree, HNN extension.
\end{abstract}

Taras Shevchenko National University of Kyiv, 64/13 Volodymyrska str., 01601, Kyiv, Ukraine

E-mail: proveronika145@gmail.com

\section{Introduction}

In recent decades groups defined by automata took a notable place among important classes of groups. Introduced in the middle of the 20th century they serve as a basement for brilliant properties and examples connecting various areas of mathematics (e.g. [2, 4,7]). Algebraic properties of these groups are diverse and they are a constant object of study.

One of the important research directions is formed by the question of which groups can be generated using automata over finite alphabets. These groups are called groups of automaton permutations and they can be viewed as automorphism groups of regular rooted trees. Residual finitness is the most valuable necessary condition for a group of automaton permutations. Another natural condition is finitness of automata that define required groups.

We consider the group $G A(\mathrm{X})$ of all automaton permutations over an alphabet $\mathrm{X}$ of prime cardinality $p$. The group $G A(\mathrm{X})$ is a profinite group and contains a naturally defined $p$-Sylow subgroup $\operatorname{Syl}_{p}(G A(\mathrm{X}))$. The latter group contains a countable subgroup $p-F G A(\mathrm{X})$ of automaton permutations defined by finite automata. The group $p-F G A(\mathrm{X})$ can be considered as the finite state wreath power of the regular cyclic group of order $p$ (see [5]). Each countable residually $p$-finite group admits an isomorphic embedding into the group $\operatorname{Syl} p(G A(\mathrm{X}))$. The main goal of the paper is to find HNN extensions of free abelian groups that admit such an embedding into the group $p-F G A(\mathrm{X})$.

We consider ascending HNN extension of free abelian groups $\mathbb{Z}^{n}, n \geq 1$. Each extension of this kind is defined by an invertible integer matrix $M$ of size $n$. We give a sufficient condition on $M$ for which the corresponding extension admit an isomorphic embedding into the group of $p-F G A(\mathrm{X})$. We give a construction of embedding that is strongly based on automata constructed in [1]. As an interesting corollary we obtain that corresponding HNN extensions are residually $p$-finite. For our best knowledge residually $p$-finite ascending HNN extension of 
free abelian groups are described only for the case $n=1$ (see [3] and references therein). Isomorphic embeddings of these groups into $p-F G A(\mathrm{X})$ were constructed in [1]. It would be interesting to find for $n>1$ sufficient conditions and even criteria on $M$ for existence of such an embedding.

The paper is organized as follows. In Section 1 we briefly recall the required definitions from automata theory and theory of groups defined by automata. Rooted trees, groups of their automorphisms and their connections with groups defined by automata are considered in Section 2. Our main constructions and results are presented in Section 3.

\section{Automata and automaton permutations}

Let $X$ be a finite set called an alphabet, $|X| \geq 2$. A finite (infinite) sequence of elements from the alphabet $X$ is called finite (infinite) word over $X$. By $X^{*}$ we denote the set of all finite words over $X$, including the empty word $\Lambda$. For the set of all infinite words over $X$ notation $X^{\omega}$ is used. By $|w|$ we denote the length of a finite word $w$. Let $X^{(n)}$ be the set of all words of length not greater than $n$ :

$$
\mathrm{X}^{(n)}=\bigcup_{0 \leq i \leq n} \mathrm{X}^{i}, \quad n \geq 0 .
$$

The set $X^{*}$ with respect to concatenation of words forms a free monoid with basis $X$. Also, concatenation of infinite words to finite words from the right is well defined. Similarly, one can define concatenation of countable sequence of finite words. In both cases the result is an infinite word.

An arbitrary finite word $w \in \mathbf{X}^{*}$ of length $l=|w|$ can be decomposed in the form

$$
w=w[1] w[2] \ldots w[l]
$$

where $w[i]$ denotes the $i$ th letter of the word $w, 1 \leq i \leq l$.

For the word $w \in \mathbf{X}^{*}$ we will use notation $\bar{w}=(w[1], w[2], \ldots, w[l])$ for the corresponding vector of length $l$.

Definition 1. An automaton $\mathcal{A}$ over alphabet $\mathrm{X}$ is a triple $\langle Q, \lambda, \mu\rangle$ such that

- $Q$ is a non-empty set, that is called the set of inner states of $\mathcal{A}$;

- $\lambda: Q \times X \rightarrow Q$ is the transition function;

- $\mu: Q \times X \rightarrow X$ is the output function.

An automaton is called finite, if the set of its inner states is finite.

For each state $q \in Q$ of the automaton $\mathcal{A}=\langle Q, \lambda, \mu\rangle$ we define the transformation $\mu_{q}: \mathrm{X} \rightarrow \mathrm{X}$ by the equality $\mu_{q}(x)=\mu(q, x), x \in \mathrm{X}$. The output function will be uniquely defined upon for each state $q \in Q$ the function $\mu_{q}$ is defined.

Definition 2. An automaton $\mathcal{A}$ is called permutational, if for each state $q$ of $\mathcal{A}$ the transformation $\mu_{q}$ is a permutation on the set $\mathrm{X}$.

In this paper we will consider only permutational automata. 
Let $\mathcal{A}=\langle Q, \lambda, \mu\rangle$ be a permutational automaton over alphabet $\mathrm{X}$. The output and transition functions $\lambda$ and $\mu$ of the automaton $\mathcal{A}$ can be naturally extended to the set $Q \times \mathrm{X}^{*}$. Namely, for arbitrary $x \in \mathrm{X}, q \in Q, w \in \mathrm{X}^{*}$ we have

$$
\begin{array}{ll}
\lambda(q, \Lambda)=q, & \lambda(q, x w)=\lambda(\lambda(q, x), w), \\
\mu(q, \Lambda)=\Lambda, & \mu(q, x w)=\mu(q, x) \mu(\lambda(q, x), w) .
\end{array}
$$

In each state $q \in Q$ of the automaton $\mathcal{A}$ the permutation on the set of all finite words $\mathrm{X}^{*}$ is defined. This permutation is called automaton permutation defined by the automaton $\mathcal{A}$ in its state $q$ and denoted by the same symbol $q$. Since the length of the common prefix of words is preserved under the action of $q$ it can be uniquely extended to the set $X^{\omega}$. The automaton permutation $q \in Q$ acts on infinite words by the following recursive rule

$$
q(u w)=\mu_{q}(u) \lambda(q, u)(w)
$$

where $u \in \mathbf{X}^{\star}, w \in \mathbf{X}^{\omega}$.

An automaton permutation over $\mathrm{X}$ is a permutation on the set $\mathrm{X}^{*}$, defined by some automaton $\mathcal{A}$ over $\mathrm{X}$ in some its state. The automaton permutation is called finite state automaton permutation if it is defined by a finite automaton.

By $G A(\mathrm{X})$ we denote the set of all automaton permutations over $\mathrm{X}$. With respect to composition $G A(\mathrm{X})$ forms a group. The set $F G A(\mathrm{X})$ of all finite state automaton permutations over $\mathrm{X}$ is a subgroup of $G A(\mathrm{X})$. For an automaton $\mathcal{A}$ over $\mathrm{X}$ the group of the automaton $\mathcal{A}$ is the subgroup of $G A(\mathrm{X})$ generated by all automaton permutations, defined in inner states of $\mathcal{A}$. The group of the automaton $\mathcal{A}$ is denoted by $G(\mathcal{A})$.

\section{Rooted trees and their automorphisms}

Let $\mathrm{X}$ be an alphabet of cardinality $d, d \geq 2$. Consider a $d$-rooted tree $\mathcal{T}_{d}(\mathrm{X})$ (see Figure 1 ). The set of vertices of $\mathcal{T}_{d}(\mathrm{X})$ is the set $\mathrm{X}^{*}$ of all finite words over $\mathrm{X}$. Two vertices $v$ and $u$ from $\mathrm{X}^{*}$ are connected by an edge if and only if $u=v x$ or $v=u x$ for some $x \in \mathrm{X}$. The root of $\mathcal{T}_{d}(\mathrm{X})$ is the empty word $\Lambda$.

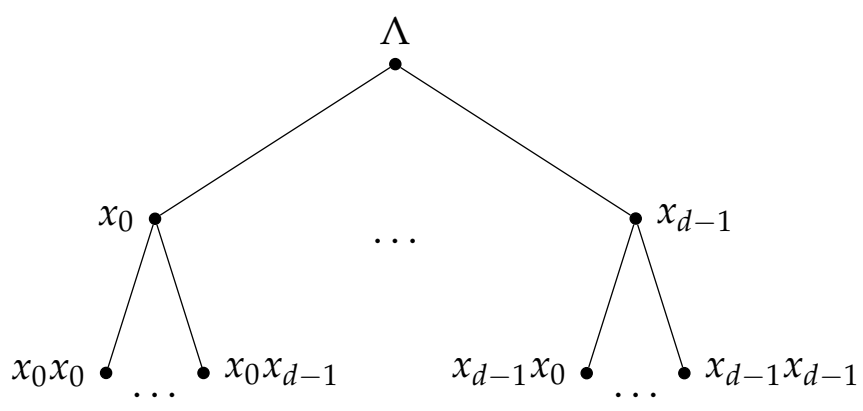

Figure 1: $d$-rooted tree $\mathcal{T}_{d}(\mathrm{X})$.

An automorphism of the tree $\mathcal{T}_{d}(\mathrm{X})$ is a bijection $f: \mathrm{X}^{*} \rightarrow \mathrm{X}^{*}$ such that $f$ preserves edges, i.e. vertices $f(v)$ and $f(v x)$ are connected for each connected vertices $v$ and $v x, v \in \mathbf{X}^{*}, x \in \mathbf{X}$. For an automorphism $f$ and a vertex $v \in X^{*}$ we consider the set of all vertices of the form $v x, x \in \mathrm{X}$. Then the permutation $\sigma_{v} \in S(\mathrm{X})$, such that $g(v x)=g(v) \sigma_{v}(v), x \in \mathrm{X}$, is well defined. 
For an automorphism $f$ let us consider the labeled tree $\mathcal{T}_{d}(\mathrm{X})$ such that every vertex $v \in \mathrm{X}^{*}$ is labeled by $\sigma_{v} \in S(\mathrm{X})$. Then such labeled tree is called the portrait of the automorphism $f$. Every automorphism is uniquely defined by its portrait.

By Aut $\mathcal{T}_{d}(\mathrm{X})$ we denote the group of all automorphisms of the tree $\mathcal{T}_{d}(\mathrm{X})$. The group Aut $\mathcal{T}_{d}(\mathrm{X})$, as a group of permutations of the set $\mathrm{X}^{\star}$, coincides with the group of automaton permutations $G A(\mathrm{X})$.

A set $\mathrm{X}^{l} \subset \mathrm{X}^{*}$ is called the $l$ th level of the tree $\mathcal{T}_{d}(\mathrm{X}), l \geq 0$. For each $n \geq 0$ we consider the rooted sub-tree of the tree $\mathcal{T}_{d}(\mathrm{X})$, such that the set of its vertices equals to the set of first $n+1$ levels of the tree $\mathcal{T}_{d}(\mathrm{X})$, i.e. to $\mathrm{X}^{(n)}$. This tree is called $d$-rooted tree of depth $n$ and denoted by $\mathcal{T}_{d, n}(\mathrm{X})$.

For each $n \geq 0$ by Aut $\mathcal{T}_{d, n}(\mathrm{X})$ we denote the group of automorphisms of the tree $\mathcal{T}_{d, n}(\mathrm{X})$. Let us define surjective homomorphisms

$$
\psi_{n}: \text { Aut } \mathcal{T}_{d, n}(\mathrm{X}) \rightarrow \text { Aut } \mathcal{T}_{d, n-1}(\mathrm{X}), \quad n \geq 1
$$

The homomorphism $\psi_{n}$ maps each automorphism $f \in$ Aut $\mathcal{T}_{d, n}(\mathrm{X})$ to its restriction on the tree $\mathcal{T}_{d, n-1}(\mathrm{X})$. Then we obtain the inverse system of finite groups Aut $\mathcal{T}_{d, n}(\mathrm{X})$ and their homomorphisms $\psi_{n}, n \geq 1$. The limit group of this inverse system is isomorphic to $G A(\mathrm{X})$. It implies that the group $G A(X)$ is profinite.

Fix a prime number $p$ and an alphabet $\mathrm{X}$ of cardinality $p$. In this case the group $G A(\mathrm{X})$ is a pro $p$-group (see [8]). The group $G A(\mathrm{X})$ contains Sylow $p$-subgroups. We denote a Sylow $p$-subgroup of $G A(\mathrm{X})$ by $\operatorname{Syl}_{p}(G A(\mathrm{X}))$. This group contains an isomorphic copy of arbitrary countable residually finite $p$-group.

We denote by $p-F G A(\mathrm{X})$ the intersection of $S y l_{p}(G A(\mathrm{X}))$ and $F G A(\mathrm{X})$. Each element of the group $\operatorname{Syl}_{p}(G A(\mathrm{X}))$ can be naturally presented by automata of special kind. Namely, fix a cycle $\sigma$ of length $p$ over $\mathrm{X}$.

Definition 3. An automaton $A=\langle Q, \lambda, \mu\rangle$ over $\mathrm{X}$ is called $p$-automaton if for each state $q \in Q$ the output function $\mu$ satisfies the equality $\mu_{q}=\sigma^{k}$ for some $k \geq 0$.

The group $\operatorname{Syl}_{p}(G A(\mathrm{X}))$ (the group $p-F G A(\mathrm{X})$ ) consists of automaton permutations that can be defined by a $p$-automaton (finite $p$-automaton) over an alphabet $\mathrm{X}$.

\section{Main results}

Let $p$ be a fixed prime, $n$ be a positive integer. Fix an integer square matrix $M=\left(m_{i j}\right)$ of size $n$ such that

(i) $m_{i i} \equiv 1(\bmod p), 1 \leq i \leq n$;

(ii) $p$ divides $m_{i j}$ for $i \neq j, 1 \leq i, j \leq n$;

(iii) no positive degree of $M$ is the identity matrix.

For instance, any matrix with positive integer elements such that each diagonal element equals 1 and all others are divisible by $p$ satisfy conditions (i)-(iii). 
Lemma 1. The determinant of the matrix $M$ is coprime with $p$.

Proof. The determinant of $M$ can be expressed in the following form

$$
\operatorname{det}(M)=m_{11} m_{22} \ldots m_{n n}+a,
$$

where $a$ is a sum of products of matrix elements such that each of them contains at least one non-diagonal element of $M$. Condition (i) implies $m_{11} m_{22} \ldots m_{n n} \equiv 1(\bmod p)$. From condition (ii) it follows that $p$ divides $a$.

Therefore, $\operatorname{det}(M) \equiv 1(\bmod p)$ and the proof is complete.

In particular, Lemma 1 implies that the matrix $M$ is non-degenerate. In fact, the proof only requires conditions (i)-(ii). However, condition (iii) does not follow from (i)-(ii).

Recall that the norm of a real valued square matrix $A=\left(a_{i j}\right)$ of size $n$ is defined as

$$
\|A\|=\max _{i} \sum_{j=1}^{n}\left|a_{i j}\right| .
$$

Lemma 2. The equality $\|M\| \geq p-1$ holds.

Proof. Let us consider two cases.

Let $M$ be a non-diagonal matrix. From condition (ii) it follows, that for each element $m_{i j} \neq 0$, which is non-diagonal in the matrix $M$, the inequality $\left|m_{i j}\right| \geq p$ holds. Then we have $\|M\| \geq p>p-1$.

Let $M$ be a diagonal matrix. From condition (iii) it follows, that $M$ is not the identity matrix. Therefore, there exists a diagonal element $m_{i i}$, such that $m_{i i} \neq 1$. Condition (i) implies inequality $\left|m_{i j}\right| \geq p-1$. Hence, $\|M\| \geq p-1$.

In both cases we have the required inequality.

Fix an alphabet $X=\{0,1, \ldots, p-1\}$ which we identify with the field of residues modulo $p$. The alphabet $X^{n}$ we identify with the vector space $V$ of dimension $n$ over field $\mathbb{Z}_{p}$.

In $[1,6]$ it was constructed the automaton $\mathcal{A}_{M}=\langle Q, \lambda, \mu\rangle$ over the alphabet $\mathrm{X}^{n}$ such that

- $Q$ is the set of vectors-columns of dimension $n$, such that each coordinate lies in the closed interval $[-\|M\|,\|M\|-1]$;

- $\lambda(v, x)=\operatorname{Div}(v+M x), v \in Q, x \in X^{n}$, where Div denotes the operation of taking coordinate-wise quotients from division by $p$;

- $\mu(v, x)=\operatorname{Mod}(v+M x), v \in Q, x \in X^{n}$, where Mod denotes the operation of taking coordinate-wise remainders from division by $p$.

Consider the group

$$
\left.G_{M}=\left\langle a_{1}, a_{2}, \ldots, a_{n}, t\right| a_{i} \text { commute, } t a_{i} t^{-1}=a_{1}^{m_{1 i}} a_{2}^{m_{2 i}} \ldots a_{n}^{m_{n i}}, 1 \leq i \leq n\right\rangle .
$$

The group $G_{M}$ is an $H N N$ extension of the free abelian group $\mathbb{Z}^{n}$.

From Lemma 1 it follows, that the group of the automaton $\mathcal{A}_{M}$ is isomorphic to the group $G_{M}$ (for the proof see [1, Proposition 4], [6, Proposition 4.7]).

Denote by $G$ the additive group of the vector space $V$. The group $G$ is isomorphic to the direct product of $n$ cyclic groups of order $p$, i.e. $\mathbb{Z}_{p}^{n}$. 
Lemma 3. 1) For arbitrary $v \in Q, x \in \mathrm{X}^{n}$, the following equality holds

$$
\mu_{v}(x)=\operatorname{Mod}(v+x) .
$$

2) In each state $v \in Q$ of the automaton $\mathcal{A}_{M}$ the function $\mu_{v}$ defines the natural action of some element of the group $G$ on $\mathbb{Z}_{p}^{n}$.

3) Each element of the group $G$ is defined by some state of the automaton $\mathcal{A}_{M}$.

Proof. Fix a state $v \in Q$.

Conditions (i) and (ii) imply that for each $x \in X^{n}$ we have equalities

$$
\begin{aligned}
\mu_{v}(x)=\operatorname{Mod}(v+M x) & =\operatorname{Mod}\left(\begin{array}{c}
v_{1}+m_{11} x_{1}+\sum_{j=2}^{n} m_{i j} x_{j} \\
\ldots \\
v_{n}+\sum_{j=1}^{n-1} m_{i j} x_{j}+m_{n n} x_{n}
\end{array}\right) \\
& =\operatorname{Mod}\left(\begin{array}{c}
v_{1}+x_{1} \\
\ldots \\
v_{n}+x_{n}
\end{array}\right)=\operatorname{Mod}(v+x) .
\end{aligned}
$$

Hence, equality (1) holds.

For $v_{1}, v_{2} \in Q, x \in X^{n}$, we have

$$
\mu_{v_{2}}\left(\mu_{v_{1}}(x)\right)=\operatorname{Mod}\left(v_{2}+\operatorname{Mod}\left(v_{1}+x\right)\right)=\operatorname{Mod}\left(v_{2}+v_{1}+x\right)=\mu_{v_{1}+v_{2}}(x)
$$

and the second statement holds.

Now, using equality (1) and Lemma 2, we immediately obtain the third statement of the lemma.

For arbitrary permutation $\sigma \in S(\mathrm{X})$ and integer vector $g=\left(g_{0}, \ldots, g_{n-1}\right)$ of length $n, n \geq 1$, we define the automaton $\mathcal{A}(\sigma, g)=\langle Q, \lambda, \mu\rangle$ over the alphabet X such that

- $Q=\left\{v: v \in \mathrm{X}^{*},|v| \leq n-1\right\}$, i.e. the set $Q$ is the set of vertices of the tree $\mathcal{T}_{d, n-1}(\mathrm{X})$;

- $\lambda(v, x)=\left\{\begin{array}{ll}v x, & \text { if }|v|<n-1, \\ v, & \text { if }|v|=n-1,\end{array} \quad v \in Q, x \in \mathbf{X}\right.$

- $\mu_{v}=\sigma^{g|v|}, v \in Q$.

From the definition of $\mathcal{A}(\sigma, g)$ the below statement directly follows.

Lemma 4. The automaton permutation defined by $\mathcal{A}(\sigma, g)$ in state $\Lambda$ maps the word $x_{1} x_{2} \ldots x_{n}$ over $\mathrm{X}$ of length $n$ to the word $\sigma^{g_{0}}\left(x_{1}\right) \sigma^{g_{1}}\left(x_{2}\right) \ldots \sigma^{g_{n-1}}\left(x_{n}\right)$.

Consider the permutation $\sigma=(0,1, \ldots, p-1)$.

Let us define the automaton $\mathcal{B}_{M}=\left\langle Q_{\star}, \lambda_{\star}, \mu_{\star}\right\rangle$ over the alphabet $\mathrm{X}$. To construct $\mathcal{B}_{M}$ we modify the automaton $\mathcal{A}_{M}=\langle Q, \lambda, \mu\rangle$. For each state $v \in Q$ of the automaton $\mathcal{A}_{M}$ we consider the automaton $\mathcal{A}(\sigma, v)=\left\langle Q_{v}, \lambda_{v}, \mu_{v}\right\rangle$ over $X$ (see Figure 2). Since the state $v$ is an integer vector of length $n$ the set of inner states $Q_{v}$ of the automaton $\mathcal{A}(\sigma, v)$ is the set of vertices of the $p$-rooted tree $\mathcal{T}_{p, n-1}(\mathrm{X})$ of depth $n-1$, i.e. $\mathbb{Z}_{p}^{(n-1)}$. 


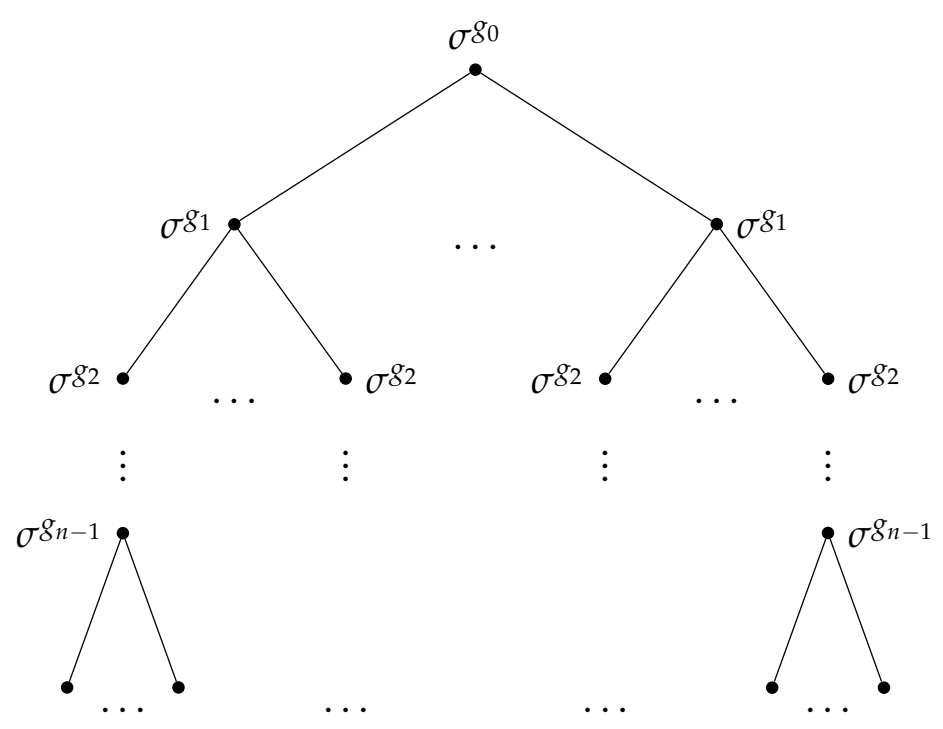

Figure 2: A portrait of the automaton permutation defined by the automaton $\mathcal{A}(\sigma, g)$ in the state $\Lambda$.

Then the automaton $\mathcal{B}_{M}$ is defined by the following equalities

- $Q_{\star}=Q \times \mathbb{Z}_{p}^{(n-1)}$;

- $\lambda_{\star}((v, w), x)=\left\{\begin{array}{ll}\left(v, \lambda_{v}(\Lambda, w x)\right), & \text { if }|w|<n-1, \\ (\lambda(v, w x), \Lambda), & \text { if }|w|=n-1,\end{array}(v, w) \in Q_{\star}, x \in \mathrm{X}\right.$

- $\mu_{\star}((v, w), x)=\mu_{v}(w, x),(v, w) \in Q_{\star}, x \in \mathrm{X}$

Let $H_{B}$ be a subgroup of the group $G\left(\mathcal{B}_{M}\right)$ generated by automaton permutations, defined in states $(v, \Lambda), v \in Q$.

Lemma 5. The group $H_{B}$ is isomorphic to the group $G_{M}$.

Proof. Consider an infinite word $w=x_{1} x_{2} \ldots x_{n} \ldots$ over $\mathrm{X}$. The word $w$ can be naturally splitted into blocks of length $n$ :

$$
\begin{aligned}
w & =\left(x_{1} x_{2} \ldots x_{n}\right)\left(x_{n+1} x_{n+2} \ldots x_{2 n}\right) \ldots\left(x_{k n+1} x_{k n+2} \ldots x_{(k+1) n}\right) \ldots \\
& =w[1, n] w[2, n] \ldots w[k, n] \ldots
\end{aligned}
$$

Define the infinite word $\varphi(w)=y_{1} y_{2} \ldots y_{k} \ldots$ over $X^{n}$, such that $y_{i}=\overline{w[i, n]}, i \in \mathbb{N}$. It is easy to see, that the mapping $\varphi$ from $X$ to $X^{n}$ is one-to-one.

For each $v \in Q$ consider states $v,(v, \Lambda)$ of the automata $\mathcal{A}_{M}$ and $\mathcal{B}_{M}$ respectively. Denote by $f_{v}$ and $F_{v}$ automaton permutations over alphabets $X^{n}$ and $X$ defined in states $v$ and $(v, \Lambda)$ respectively. To prove the lemma it is enough to show, that for arbitrary infinite word $w$ over $\mathrm{X}$ and arbitrary state $v \in Q$ the following equality holds

$$
f_{v}(\varphi(w))=\varphi\left(F_{v}(w)\right)
$$


Consider each part of equality (3). Split out an infinite word $w$ on the blocks of the length $n$ (see (2)). Then we have $w=w[1, n] w_{1}$ for some infinite word $w_{1}$ over $\mathbf{X}$.

Therefore, the following equality holds

$$
\begin{aligned}
F_{v}(w)=F_{v}\left(w[1, n] w_{1}\right) & =\mu_{\star}((v, \Lambda), w[1, n]) F_{\lambda(v, w[1, n])}\left(w_{1}\right) \\
& =\mu_{v}(\Lambda, w[1, n]) F_{\lambda(v, w[1, n])}\left(w_{1}\right) .
\end{aligned}
$$

Moreover, by the definition of the automaton $\mathcal{A}_{M}$ the state $v \in Q$ is an integer vector $\left(v_{0}, v_{1}, \ldots, v_{n-1}\right)$. Then, by Lemma 4 the following equality holds

$$
\mu_{v}(\Lambda, w[1, n])=\sigma^{v_{0}}\left(x_{1}\right) \sigma^{v_{1}}\left(x_{2}\right) \ldots \sigma^{v_{n-1}}\left(x_{n}\right)
$$

Finally, we have

$$
F_{v}(w)=\sigma^{v_{0}}\left(x_{1}\right) \sigma^{v_{1}}\left(x_{2}\right) \ldots \sigma^{v_{n-1}}\left(x_{n}\right) F_{\lambda(v, w[1, n])}\left(w_{1}\right)
$$

On the other hand, by the definition of automaton permutation, the left part of equality (3) can be rewritten in the following form

$$
f_{v}(\varphi(w))=f_{v}\left(\overline{w[1, n]} \varphi\left(w_{1}\right)\right)=\mu_{v}(\overline{w[1, n]}) f_{\lambda(v, \overline{w[1, n]})}\left(\varphi\left(w_{1}\right)\right)
$$

Then by Lemma 3 we have

$$
\mu_{v}(\overline{w[1, n]})=\operatorname{Mod}(v+\overline{w[1, n]})=\left(\sigma^{v_{0}}\left(x_{1}\right), \sigma^{v_{1}}\left(x_{2}\right), \ldots, \sigma^{v_{n-1}}\left(x_{n}\right)\right)
$$

Therefore, from equalities (4)-(6) it follows the required equality (3) and the statement of the lemma is proved.

Now we can formulate the main theorem.

Theorem 1. The group $G_{M}$ is isomorphic to a subgroup of $p-F G A(X)$.

Proof. Let $q$ be an arbitrary state of the automaton $\mathcal{B}_{M}$. Then by the definition of $\mathcal{B}_{M}$ the permutation over $\mathrm{X}$ defined by state $q$ is a power of the permutation $\sigma=(0,1, \ldots, p-1)$. Hence, the automaton $\mathcal{B}_{M}$ is a $p$-automaton.

By Lemma 5 the group $G_{M}$ is generated by automaton permutations, defined in states of the finite $p$-automaton $\mathcal{B}_{M}$. Therefore, we have the statement of the theorem.

Corollary 1. The group $G_{M}$ is residually $p$-finite.

Proof. Since the group $p-F G A(\mathrm{X})$ is residually $p$-finite and subgroups of residually $p$-finite groups posses this property the statement immediately follows from Theorem 1.

We constructed a group isomorphic to $G_{M}$ such that it is generated by automaton permutations defined by a proper subset of states of $\mathcal{B}_{M}$. A natural problem to describe the group $G\left(\mathcal{B}_{M}\right)$ of the automaton $\mathcal{B}_{M}$ is left open. 


\section{References}

[1] Bartholdi L., Šuniḱk Z. Some solvable automaton groups. In: Grigorchuk R., Mihalik M., Sapir M., Šuniḱk Z. (Eds.) Topological and asymptotic aspects of group theory. Contemp. Math. 2006, 394, Amer. Math. Soc., Providence, RI, 11-29. doi:10.1090/conm/394/07431

[2] Grigorchuk R.I. Degrees of growth of finitely generated groups, and the theory of invariant means. Math. USSR Izv. 1985, 25 (2), 259-300. doi:10.1070/IM1985v025n02ABEH001281

[3] Moldavanskii D. On the residual properties of Baumslag-Solitar groups. Comm. Algebra 2018, 46 (9), $3766-3778$. doi:10.1080/00927872.2018.1424867

[4] Nekrashevych V. Palindromic subshifts and simple periodic groups of intermediate growth. Ann. of Math. 2018, 187 (3), 667-719. doi:10.4007/annals.2018.187.3.2

[5] Oliynyk A. S. Finite state wreath powers of transformation semigroups. Semigroup Forum 2011, 82, $423-436$. doi:10.1007/s00233-011-9292-z

[6] Šunić Z., Ventura E. The conjugacy problem in automaton groups is not solvable. J. Algebra 2012, 364, 148-154. doi:10.1016/j.jalgebra.2012.04.014

[7] Sushchansky V.I. Periodic p-groups of permutations and the unrestricted Burnside problem. Dokl. Akad. Nauk SSSR 1979, 247 (3), 557-561.

[8] Wilson J.S. Profinite groups. Clarendon Press, Oxford, 1998.

Received 24.04.2021

Прохорчук В. Про скінченно станові автоматні дї HNN розиирень вільних абелевих груп // Карпатські матем. публ. - 2021. - Т.13, №1. - С. 180-188.

Розглянуто HNN розширення вільних абелевих груп. $\Delta л я$ довільного простого $p$ визначено клас таких розширень, які діють скіченно автоматними підстановками над алфавітом $X$ потужності $p$ і належать $p$-підгрупі Силова групи автоматних підстановок над $\mathrm{X}$. Як наслідок звідси випливає, шо всі відповідні HNN розширення резидуально $p$-скінченні.

Ключові слова і фрази: група автомата, автоморфізм кореневого дерева, HNN розширення. 